\title{
Impact of inertia weight strategies in particle swarm optimization for solving economic dispatch problem
}

\author{
Mohammed Amine Meziane, Youssef Mouloudi, Bousmaha Bouchiba, abdellah Laoufi \\ Tahri Mohammed University, BP 417, 08000 Bechar, Algeria
}

\begin{abstract}
Article Info
Article history:

Received Aug 3, 2018

Revised Sep 19, 2018

Accepted Oct 1, 2018

\section{Keywords:}

Convergence

Data From SONELGAZ

Economic Dispatch

Inertia Weight

Particle Swarm Optimization

ABSTRACT

Particle Swarm Optimization (PSO) is a population based stochastic optimization technique inspired by the social learning of birds or fish. Some of the appealing facts of PSO are its convenience, simplicity and easiness of implementation requiring but few parameters adjustments. Inertia Weight $(\omega)$ is one of the essential parameters in PSO, which often significantly the affects convergence and the balance between the exploration and exploitation characteristics of PSO. Since the adoption of this parameter, there have been large proposals for determining the value of Inertia Weight Strategy. In order to show the efficiency of this parameter in the Economic Dispatch problem(ED), this paper presents a comprehensive review of one or more than one recent and popular inertia weight strategies reported in the related literature. Among this five recent inertia weight four were randomly chosen for application and subject to empirical studies in this research, namely, Constant $(\omega)$, Random $(\omega)$, Global-Local Best $(\omega)$, Linearly Decreasing $(\omega)$, which are then compared in term of performance within the confines of the discussed optimization problem. Morever, the results are compared to those reported in the recent literature and data from SONELGAZ. The study results are quite encouraging showing the good applicability of PSO with adaptive inertia weight for solving economic dispatch problem.
\end{abstract}

Copyright (C) 2019 Institute of Advanced Engineering and Science. All rights reserved.

\section{Corresponding Author:}

Mohammed Amine Meziane

Tahri Mohammed University,

BP 417, 08000 Bechar, Algeria.

Email: aminemohmeziane@gmail.com

\section{INTRODUCTION}

Amongst the different issues of power systems operation, the economic load dispatch (ELD) problem is one of the key tools in operating and planning of modern electric utility grid. Essentially, electrical grid systems are interconnected and consist of power generating, transmission and distribution utilities in order to produce electrical power to consumers, at a low production cost, maximum reliability and better operating conditions. The ELD is a static problem, it was first discussed by Carpentier in 1962 [1], the main purpose of ELD is to find the optimal output power of generators to minimize the total generation cost and satisfy the equality and inequality constraints. To solve this problem many efforts have been made over the years, various mathematical programming and optimization techniques were used. A survey of literature on the methods proposed to solve ELD, which can be divided into two categories, the classic (traditional) methods and the smart (heuristic) methods. It is observed that the traditional methods and heuristic methods have some limitations to solve ELD problems. The traditional methods suffer with large execution time and would not be useful when the cost functions are nonlinear. So in some cases, it will be very difficult to achieve optimal solutions. For this reason, recently, the heuristic methods have been used to overcome this problem [2-3]. Therefore in recent years, different smart and innovative algorithms such as: Genetic Algorithm (GA) [4], Particle Swarm Optimization (PSO) [5-6], Evolutionary Programming Algorithm (EP) [7], Cuckoo Search (CS) [8] , .. have been proposed to solve this problem. 
Recently, many researches have been directed towards the application of particle swarm optimization technique to solve ELD problem [9]. The most important advantages of the PSO are that PSO is easy to implement and there are few parameters to adjust. In this article, an attempt has been made to solve economic load dispatch problem using particle swarm optimization by means of minimization of fuel costs while satisfying physical and operational limitations. However, the prominent model to be discussed in this paper, are Inertia Weight Strategies, and their effect in PSO for solving the ELD. In order to further illustrate the effect of such mechanism in PSO for solving ELD, different inertia weight mechanism is reviewed and experiments are carried out over single objective minimization case in the Real West Algeria 22-bus system to compare different strategies of setting Inertia Weight. Moreover, the obtained optimal results also compared with the some reported result found in literature and with Data from SONELGAZ. It found that the PSO capable to obtain lowest cost as compared to others. Thus, it has great potential to be implemented in different types of power system optimization problem.

\section{PARTICLE SWARM OPTIMIZATION}

PSO is a population-based optimization technique which was first introduced by Kennedy and Eberhart in 1995 [10], inspired by social behavior of bird flocking or fish schooling in search of food. PSO compared to other existing heuristic optimization strategies such as genetic algorithm, is easier to implement involving only few parameters to adjust with accurate results in term of calculus. In a PSO system, particles fly around in a multidimensional search space.

During flight, each particle adjusts its trajectory towards its own previous best position (This value is called Pbest), and towards the best previous position attained by any member of its neighborhood or globally, the whole swarm (This value is called Gbest), [11-17]. The two equations which are used in PSO are velocity update equation (1) and position update equations (2). These are to be modified at each time step, of PSO algorithm to converge the optimum solution.

$$
\begin{aligned}
& V_{i}(t+1)=\omega V_{i}(t)+c_{1} r_{1}\left[\text { Pbest }_{i}(t)-X_{i}(t)\right]+c_{2} r_{2}\left[\text { Gbest }_{i}(t)-X_{i}(t)\right. \\
& X_{i}(t+1)=X_{i}(t)+V_{i}(t+1)
\end{aligned}
$$

Where ${ }^{i}:$ is the particle index; ${ }^{\omega}$ : is the inertia coefficient; ${ }^{c 1}, c_{2}$ are acceleration coefficients

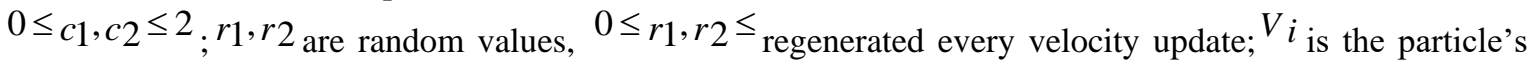
velocity at time t; $X_{i}$ is the particle's position at time t; Pbest is the particle's individual best solution as of time $\mathrm{t}$; Gbest is the swarm's best solution as of time t.

Since 1995 many attempts have been made to improve the performance of the original PSO. For instance, the maximum velocity $V \max$ was introduced to arbitrarily limit the velocities of the particles and improve the result of the search. The inertia weight $(\omega)$ is one of PSO parameters originally proposed by Shi and Eberhart [18] to bring about a balance between the exploration and exploitation characteristics of PSO. Since the introduction of this parameter, there have been a number of proposals of different strategies for determining the value of inertia weight during a course of run.

\section{DIFFERENT INERTIA WEIGHT ADAPTATION MECHANISMS}

The balance between global and local search throughout the course of a run is critical to the success of an optimization algorithm [19]. Inertia Weight plays a key role in the process of balance between the exploration and exploitation characteristics of PSO. In 1998 Shi and Eberhart [18] presented for the first time the concept of inertia weight by introducing Constant Inertia Weight in which the velocity of each particle is updated according to the equation (1). They claimed that a large inertia weight facilitates a global search while a small Inertia Weight facilitates a local search. The following paragraphs represent a review of various inertia weights in PSO chronologically.

Shi and Eberhart [20] proposed a Constant value of Inertia Weight and experimentally show that w from $[0.8,1.2]$ PSO provide the global optimum in a reasonably of iteration. The Random Inertia Weight strategy [21] is used in dynamic environment to enable PSO to track the optima and increases the convergence of the algorithm in early iterations. 


$$
\omega=0.5+\frac{\operatorname{rand}()}{2}
$$

Where $\operatorname{rand}^{()}$is a random number in $[0,1] ; \omega$ is then a uniform random variable in the range $[0.5,1]$.

In Time Varying Inertia Weight Strategies [22] the value of $\omega$ is determined based on iteration number. These methods can be either a linear or non-linear and increasing or decreasing.

A linearly Decreasing Inertia Weight [23-25] was introduced to improve the performance of PSO. They suggest that with a $\omega$ from the range 0.9 to 0.4 the PSO provides excellent results. In this method, the value of inertia weight was decreased from $\left(\omega_{\max }\right)_{\text {to }}\left(\omega_{\min }\right)$ according to the following equation:

$$
\omega(\text { iter })=\frac{\text { iter }_{\max }-\text { iter }}{\text { iter }_{\max }}\left(\omega_{\max }-\omega_{\min }\right)+\omega_{\min }
$$

Where iter $_{\text {the current iteration of the algorithm and }}$ iter $\max _{\text {is }}$ the maximum number of iterations.

In [26], Global-Local Best Inertia Weight is proposed by Arumugam and Rao. They use the ratio of the local best and global best of the particles in each generation to determine the adaptive inertia weight in each iteration.

$$
\omega=1.1-\frac{\text { gbest }}{\left(\text { pbest }_{i}\right)_{\text {average }}}
$$

Feng et al. [27-28] proposed Chaotic Inertia Weight using the merits of chaotic optimization. It found that the CRIW enhances the performance of PSO in comparison with RIW. The proposed $\mathrm{w}$ is as follows:

$$
\omega(\text { iter })=\left(\omega_{\max }-\omega_{\min }\right) \times \frac{\text { iter } \max }{\text { iter } \max }+\omega_{\min } \times z
$$

\begin{tabular}{|c|c|c|c|}
\hline No. IWS & NAME OF INERTIA WEIGHT STRATEGIES & Formula of inertia weight & Reference \\
\hline$\omega 1$ & Constant inertia weight & $\omega=c$ & [20] \\
\hline$\omega_{2}$ & Random inertia weight & $\omega=0.5+\frac{\operatorname{rand}()}{2}$ & [21] \\
\hline$\omega 3$ & Linear Decreasing inertia weight & $\omega($ iter $)=\frac{\text { iter } r_{\max }-i \text { iter }}{\text { iter } \max }\left(\omega_{\max }-\omega_{\min }\right)+\omega_{\min }$ & [23-25] \\
\hline$\omega 4$ & Global-Local Best inertia weight & $\omega=1.1-\frac{\text { gbest }}{\left(\text { pbest }_{i}\right)_{\text {average }}}$ & [26] \\
\hline$\omega 5$ & Chaotic inertia weight & $\begin{array}{c}\omega(\text { iter })=\left(\omega_{\max }-\omega_{\min }\right) \times \frac{\text { iter } \max -\text { iter }}{\text { iter } \max _{\max }}+\omega_{\min } \times z \\
z=4 \times z \times(1-z)\end{array}$ & [27-28] \\
\hline
\end{tabular}

The summary of various inertia weight strategies are displayed in Table 1.

Table 1. Different Inertia Weight Adaptation Strategies

\section{OBJECTIVE}

The inertia weight strategies have been suggested to improve both exploration and exploitation ability or one of them in PSO. Exploitation means that all particles converge to the same peak of the objective function and remains there. Furthermore, the exploration characteristic shows the capability of the algorithm to leave the current peak and looking for better solutions.

Considering the above clarifications, the investigator aim at exploring the impact of inertia weight on the exploration and exploitation capabilities in PSO and suggest a better strategy for users of this algorithm within the area of the ELD problem. Experiments have been carried out on four Inertia Weight Strategies: Constant $(\omega)$, Random $(\omega)$, Global-Local Best $(\omega)$, Linearly Decreasing $(\omega)$ in the confine of economic dispatch optimization problem for 22 bus in power network real, West Algeria.

Impact of inertia weight strategies in particle swarm optimization for ... (Mohammed Amine Meziane) 


\subsection{Economic Dispatch}

The economic dispatch problem, which is used to minimize the cost of production of real power, can generally be stated as follows:

$$
\begin{aligned}
& \operatorname{Min}\left[\sum_{i=1}^{n} F_{i}\left(P_{i}\right)\right] \\
& \sum_{i=1}^{n} P_{i}=P D^{-} P_{L} \\
& P_{n}^{\min } \leq P_{i} \leq P_{n}^{\max }
\end{aligned}
$$

Where, generally, $F_{i}\left(P_{i}\right)$ is a quadratic curve;

$$
F_{i}\left(P_{i}\right)=a_{i} P_{i}^{2}+b_{i} P_{i}+c_{i}
$$

Here:

$a_{i}, b_{i}$ and $^{c i}$ are the known coefficient; ${ }^{n}$ : number of generators; $P i$ : real power generation; $P D:$ real power load; $P L$ : real losses.

\subsection{Experiment procedures}

In order to test and compare some different inertia weight strategies in PSO reviewed in this research, important optimization problem such as static economic dispatch for 22 bus in power network real, West Algeria are used. Inertia weight mechanism's influence on the ELD problem is tested in terms of convergence speed and solution quality in the PSO algorithm.

The parameters settings of the experiment are as follows:

Population size (Swarm size) is 100 particles. The maximum iteration allowed number of function evaluations is 200. The value of acceleration parameters $\mathrm{c} 1$ and $\mathrm{c} 2$ are taken equal to 2 . The experiment conducted in the ELD investigation was set in 22 bus system of power network real, West Algeria. This latter consists of 7 thermal units, 15 load buses and 31 transmission lines, 03 compensator var static SVC [3* (+40Mvar et )10Mvar)]. The total system demand is $856 \mathrm{MW}$. For implementing these different strategies in PSO, the programming of the ELD problem using the PSO method has been developed and applied using MATLAB software environment, tested on a CORE i5, personal computer with $2.20 \mathrm{GHz}$ and 4 GO RAM.

\section{SIMULATION RESULT AND DISCUSSION}

The four strategies adopted for comparisons: Constant Inertia Weight, Random Inertia Weight, Global-Local Best Inertia Weight and Linear Decreasing Inertia Weight are shown in Table 2 providing the best solutions of the ELD problem.

Table 2. Optimization Results of Diffrent Inertia Weight Strategies in PSO for Economic Dispatch

\begin{tabular}{ccccc}
\hline Criterion & Constant & Random & Global-Local Best & $\begin{array}{c}\text { Linear Decreasing } \\
\omega\end{array}$ \\
\hline$P_{1}[\mathrm{MW}]$ & 320 & 320 & 320 & 182.826 \\
$P_{2}[\mathrm{MW}]$ & 140 & 140 & 140 & 192.257 \\
$P_{3}[\mathrm{MW}]$ & 100 & 102.5166 & 100.6703 & 154.319 \\
$P_{4}[\mathrm{MW}]$ & 104.6458 & 102.0303 & 103.9486 & 150 \\
$P_{5}[\mathrm{MW}]$ & 110 & 110 & 110 & 63.7198 \\
$P_{6}[\mathrm{MW}]$ & 50 & 50 & 50 & 50 \\
$P_{7}[\mathrm{MW}]$ & 80 & 79.9998 & 80 & 79.9986 \\
Transmission Loss & 48.6458 & 48.5482 & 48.6189 & $\mathbf{1 7 . 1 2}$ \\
Total output & 904.6458 & 904.5482 & 904.6189 & 873.1204 \\
Load demand & 856 & 856 & 856 & 856 \\
Total Cost $[\$ \mathrm{~h}]$ & 9548.9 & 9549 & 9548.9 & $\mathbf{8 9 9 9 . 3 4}$ \\
\hline
\end{tabular}


According to the above table, we notice that Constant and Global-Local Best Inertia Weight gives us the same production cost and a slightly lower of $0.1 \$ / \mathrm{h}$ in comparison with Random Inertia Weight, transmission losses given by Random $(\omega)$ is lower than that given by Constant $(\omega)$ and Global-Local Best $(\omega)$. In contrast, a Linearly Decreasing Inertia Weight gives a much better production cost of 549.66 [\$/h] and minimum transmission loss of 31.5258 [MW], in comparison to other strategies. The difference in generation cost between these mechanisms and in real power loss clearly shows the advantage of this mechanism. Figure 1 illustrates convergence characteristics of PSO using the four Inertia Weight Strategies.

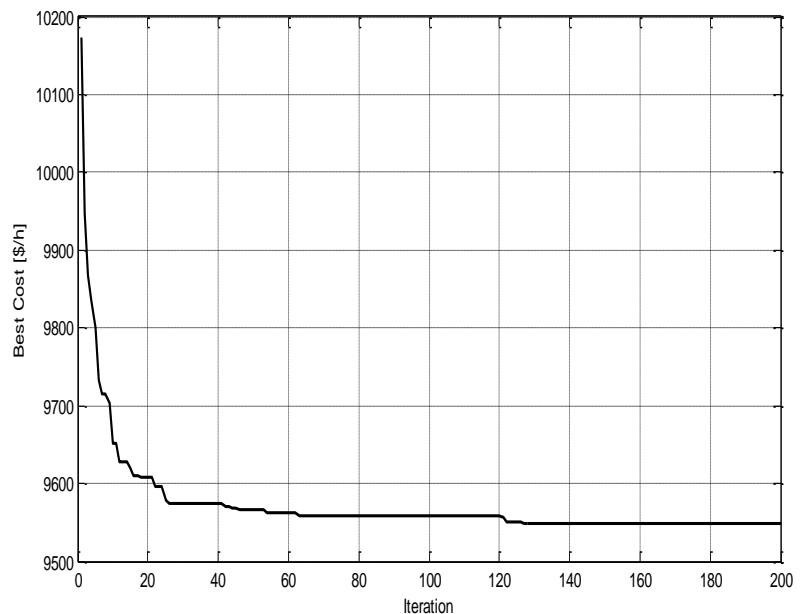

(a)

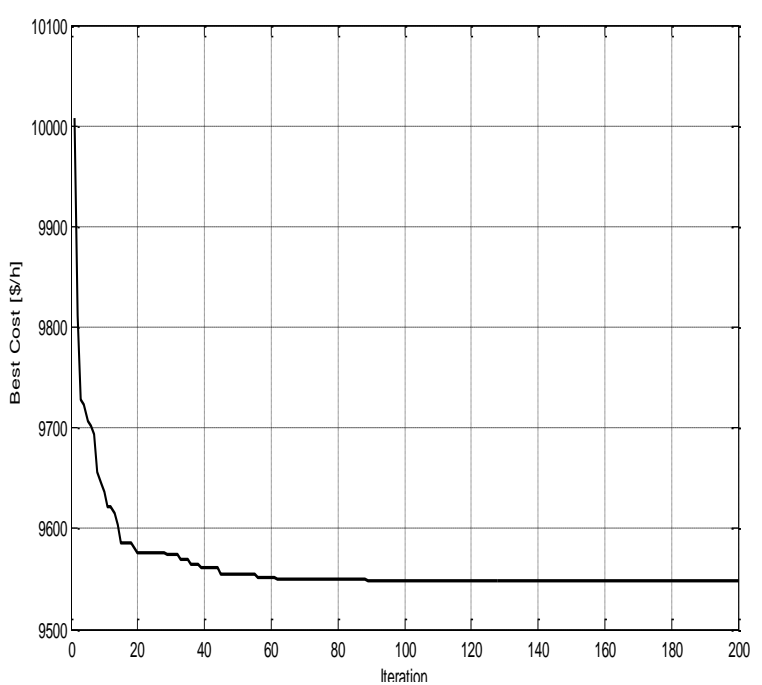

(c)

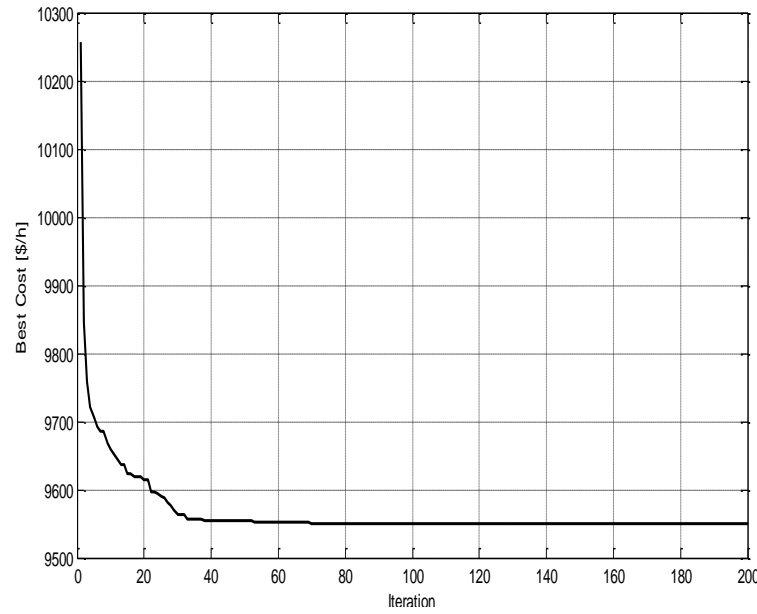

(b)

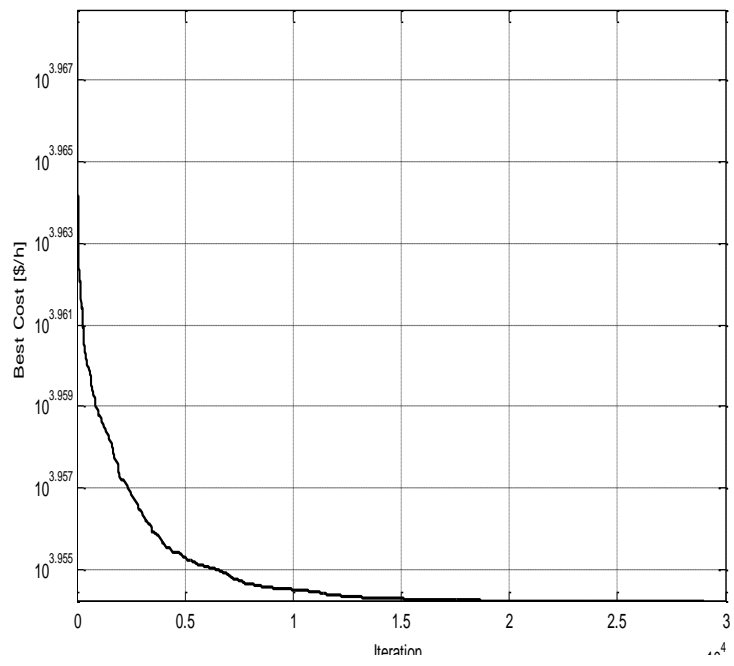

(d)

Figure 1. Convergence characteristic of PSO using four inertia weight adjusting methods on (a) Constant ( $\omega)$;

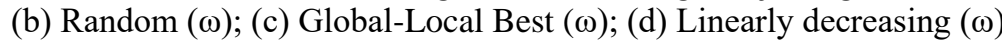

These graphs clearly indicate that PSO converges rapidly to a high quality solution at the early iterations. The minimize cost and power loss obtained by the proposed algorithm is less than value reported in [29-31] using the evolutionary copulation techniques, genetic algorithm, Ant colony optimization for the some test systems.

In order to demonstrate the efficiency and the robustness of the proposed PSO and the performance of usage the inertia weight strategie in PSO for the solution of economic dispatch. The results obtained for the power network real, West Algeria $220 \mathrm{kV}$ of the 22-bus are compared to those obtained using Data from SONELGAZ and present in Table 3. 


\begin{tabular}{lcl} 
& Table 3. Comparison Results \\
\hline \multicolumn{1}{c}{ Criterion } & Data From SONELGAZ & Linear Decreasing $\omega$ \\
\hline$P_{1}[\mathrm{MW}]$ & 200 & 182.826 \\
$P_{2}[\mathrm{MW}]$ & 200 & 192.257 \\
$P_{3}[\mathrm{MW}]$ & 300 & 154.319 \\
$P_{4}[\mathrm{MW}]$ & 80 & 150 \\
$P_{5}[\mathrm{MW}]$ & 100 & 63.7198 \\
$P_{6}[\mathrm{MW}]$ & 100 & 50 \\
$P_{7}[\mathrm{MW}]$ & 10 & 79.9986 \\
Transmission Loss & 21.40 & 17.12 \\
Total output & 890 & 873.1204 \\
Load demand & 856 & 856 \\
Total Cost $[\$ / \mathrm{h}]$ & 9104.42 & 8999.34 \\
\hline
\end{tabular}

From the above table, it appears that PSO algorithm when using Linearly Decreasing Inertia Weight gives much better results than the Data from Sonelgaz. The difference in generation cost and in Real power loss clearly shows the advantage of this method.

\section{CONCLUSION}

In this paper, a comparative study on four suggested inertia weight strategies was conducted to improve their impact on exploration and exploitation abilities in particle swarm optimization algorithm over economic dispatch problem. These strategies are Constant Inertia Weight, Random Inertia Weight, GlobalLocal Best Inertia Weight and Linear Decreasing Inertia Weight. The results verified and proved the main objective of this study about the impact of inertia weight on the performance of PSO for optimal dispatch. As an overall outcome of the experiments results carried out assignment, Linear Decreasing Inertia Weight is the best strategy for a better production cost and a low transmission losses.

\section{ACKNOWLEDGMENT}

Authors would like to thank the heads of Laboratory of Analysis, Control and Optimization of Electro-Energetic Systems (CAOSEE) and Smart Grids the renewable energies (ENERGARID) at the university TAHRI Mohammed of Bechar (Algeria).

\section{REFERENCES}

[1] Hongye Wang, Carlos E. Murillo-Sanchez, Ray D. Zimmerman and Robert J. Thomas. On Computational Issues of Market-Based Optimal Power Flow. IEEE Transactions on Power Systems, Aug 2007 ; Vol. 22(3) : 1185-1193.

[2] G.Sreenivasan, Dr. C.H.Saibabu, Dr.S.Sivanagaraju. Solution of Dynamic Economic Load Dispatch (DELD) Problem with Valve Point Loading Effects and Ramp Rate Limits Using PSO. International Journal of Electrical and Computer Engineering (IJECE). 2011; Vol.1 (1):59-70.

[3] Hossein Shahinzadeh, Sayed Mohsen Nasr-Azadani, Nazereh Jannesari, "Applications of Particle Swarm Optimization Algorithm to Solving the Economic Load Dispatch of Units in Power Systems with Valve-Point Effects", International Journal of Electrical and Computer Engineering (IJECE). 2014; Vol. 4, pp. 858 867.

[4] Walter DC, Sheble GB. Genetic algorithm solution of economic dispatch with valve point loading. IEEE Transactions on Power Systems. 1993; 8(3): 1325-1332.

[5] Jong-Bae Park, Ki-Song Lee, Joong-Rin Shin, Lee KY. A particle swarm optimization for economic dispatch with non-smooth cost functions. IEEE Transactions on Power Systems. 2005; 20(1): 34-42.

[6] Jiang S, Ji Z, Shen $Y$, “A novel hybrid particle swarm optimization and gravitational search algorithm for solving economic emission load dispatch problems with various practical constraints”, Int J Electr Power Energy Syst, 2014; 55: 628-44.

[7] Venkatesh, P Gnanadass R, Padhy NP. Comparison and application of evolutionary programming techniques to combined economic emission dispatch with line flow constraints. IEEE Transactions on Power Systems. 2003; 18(2): 688-697.

[8] N. Karthik, A.K. Parvathy RA, "Non-convex Economic Load Dispatch using Cuckoo Search Algorith". Indonesian Journal of Electrical Engineering and Computer Science (IJEECS), 2017; 5: 48-57.

[9] R. Chakrabarti, P K Chattopadhyay, M Basu, C K Panigrahi, "Particle Swarm Optimization Technique For Dynamic Economic Dispatch", July 26, 2005.

[10] J. Kennedy, R.C. Eberhart, Particle swarm optimization. IEEE International Conference on Neuran Networks.1995; pp :1942-1948. 
[11] Djilani Ben Attous, Yacine Labbi. Particle Swarm Optimization based Optimal Power Flow for Units with NonSmooth Fuel Cost Functions. International Conference on Electrical and Electronics Engineering. Bursa, Turkey. $2009: 377-381$.

[12] Eberhart, R.C., and Shi, Y. Comparing inertial weights and Constriction factor in particle Swarm optimization. proceeding of the 2000 International Congress on Evaluating Computation, San Diego, California. IEEE Service Center, Piscataway; 2000: 84-88.

[13] Sajjad Ahmadnia, Ehsan Tafehi. Using Particle Swarm Optimization, Genetic Algorithm, Honey Bee Mating Optimization and Shuffle Frog Leaping Algorithm for Solving OPF Problem with their Comparison. TELKOMNIKA Indonesian Journal of Electrical Engineering. 2015. Vol. 15(3):445-451.

[14] Kennedy. J. and Eberhart, R.C. Particle Swarm Optimzation. Proceeding of the 1997 International Conference on Evaluationary Computation, IEEE service Center, Piscataway. 1997; pp: 303-308.

[15] Youssef MOULOUDI, Mohammed Amine MEZIANE, Abdellah LAOUFI,Bousmaha BOUCHIBA, Othmane HARISI. A Swarm Algorithm Intelligent Optimization PSO in Power Network Real, West Algeria $220 \mathrm{kV}$.

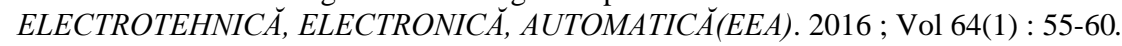

[16] J. C. Bansal, \& all. Inertia Weight Strategies in Particle Swarm Optimization. IEEE Third World Congress on Nature and Biologically Inspired Computing. Salamanca, Spain. 2011.

[17] Samar Bashath, Amelia Ritahani Ismail, Comparison of Swarm Intelligence Algorithms for High Dimensional Optimization Problems. Indonesian Journal of Electrical Engineering and Computer Science. 2018; Vol. 11(1): 300-307.

[18] Y. Shi and R. Eberhart. A modified particle swarm optimizer. In Evolutionary Computation Proceedings, 1998. IEEE World Congress on Computational Intelligence. 2002 ; pages 69-73.

[19] Y. Shi, R. Eberhart, Fuzzy adaptive particle swarm optimization. In Congress on Evolutionary Computation. Seoul, Korea ; 2001.

[20] R.C. Eberhart and Y. Shi. Tracking and optimizing dynamic systems with particle swarms. Proceedings of the 2001 Congress on Evolutionary Computation. 2002. Vol $1: 94-100$.

[21] Y.H. Shi, R.C. Eberhart. A modified particle swarm optimizer. in: IEEE International Conference on Evolutionary Computation, Anchorage Alaska, 1998, pp. 69-73.

[22] Ahmed Nickabadi et al. A novel particle swarm optimization algorithm with adaptive inertia weight. Applied Soft Computing. 2011: 3658-3670.

[23] J. Xin, G. Chen, and Y. Hai. A Particle Swarm Optimizer with Multistage Linearly-Decreasing Inertia Weight. International Joint Conference on Computational Sciences and Optimization. CSO 2009. Vol 1: 505-508.

[24] R.C. Eberhart, Y.H. Shi. Comparing inertia weights and constriction factors in particle swarm optimization. In: IEEE Congress on Evolutionary Computation. 2000: pp. 84-88.

[25] Y.H. Shi, R.C. Eberhart. Experimental study of particle swarm optimization. In: Conference, Orlando, 2000.

[26] M.S. Arumugam and MVC Rao. On the performance of the particle swarm optimization algorithm with various Inertia Weight variants for computing optimal control of a class of hybrid systems. Discrete Dynamics in Nature and Society, 2006.

[27] Y. Feng, G. Teng, A. Wang, Y.M. Yao. Chaotic inertia weight in particle swarm optimization. In: Second International Conference on Innovative Computing, Information and Control (ICICIC 07), 2007, pp: 475-1475.

[28] Y. Feng, Y.M. Yao, A. Wang. Comparing with chaotic inertia weights in particle swarm optimization. In: International Conference on Machine Learning and Cybernetics, August 2007. pp: 329-333.

[29] W. Ongsakul, T. Tantimaporn. Optimal powers flow by improved evolutionary programming. Elect. Power Comp. and Syst. 2006; Vol. 34: pp. 79-95.

[30] J. Yuryevich, K. P. Wong, Evolutionary Programming Based Optimal Power Flow Algorithm. IEEE Transaction on power Systems. 1999; Vol. 14, No. 4.

[31] C. Thitithamrongchai, B. Eua-arporn. Selfadaptive Differential Evolution Based Optimal Power Flow for Units with Non-smooth Fuel Cost Functions. J. Electrical Systems. 2007: 88-99. 